Revista Brasil. Bot., V.32, n.4, p.677-689, out.-dez. 2009

\title{
Distilia e homostilia em espécies de Palicourea Aubl. (Rubiaceae) do Cerrado do Brasil Central ${ }^{1}$
}

\author{
HÉLDER CONSOLARO ${ }^{2,5}$, RAFAELA DECESARE PARMEZAN TOLEDO ${ }^{3}$, \\ RAFAEL LOPEZ FERREGUTI ${ }^{3}$, JOHN HAY ${ }^{3}$ e PAULO EUGÊNIO DE OLIVEIRA ${ }^{4}$
}

(recebido: 3 de abril de 2008; aceito: 12 de agosto de 2009)

\begin{abstract}
Distyly and homostyly in species of Palicourea Aubl. (Rubiaceae) from the Cerrado of Central Brazil). Distyly is a type of reproductive system whose morphological and physiological features are used as a mechanism for increasing the xenogamy. Among the 25 Angiosperm families presenting this system, Rubiaceae is the richest. The objective of this study was to characterize the distylous system of four Palicourea species from the Cerrado of Central Brazil, Palicourea marcgravii A. St.-Hil., P. officinalis Mart., P. coriacea (Cham.) K. Schum. e P. macrobotrys (Ruiz \& Pav.) Roem. \& Schult. This study took place in the following areas of Distrito Federal: Parque Nacional de Brasília, Estação Ecológica de Águas Emendadas e Reseva Ecológica do IBGE. The species Palicourea marcgravii and P. officinalis were typicall distylous due to the presence of two floral morphs, the in ratio, and also the self and intramorph incompatibility. However, $P$. coriacea and P. macrobotrys species were variants because homostylous individuals occur together with distylous morphs in first specie, and because the homostyle morph is dominant in the second. It is still unknown which evolutional drivers determine those differences. However, it is likely that intrinsic genetics characteristics of each taxon have a considerable influence, and also that species may respond differently to the same selective pressures, or they may not present any response.
\end{abstract}

Key words - floral morphometric, heterostyly, isoplethy, pollination, reproductive biology

RESUMO - (Distilia e homostilia em espécies de Palicourea Aubl. (Rubiaceae) do Cerrado do Brasil Central). Distilia é um tipo de sistema reprodutivo que utiliza características morfológicas e fisiológicas como forma de potencializar a xenogamia. Dentre as 25 famílias de Angiospermas que apresentam esse sistema, Rubiaceae é a que tem a maior riqueza. Este estudo teve como objetivo investigar os aspectos relacionados ao sistema distílico de quatro espécies de Palicourea distribuídas no Cerrado do Brasil Central: Palicourea marcgravii A. St.-Hil., P. officinalis Mart., P. coriacea (Cham.) K. Schum. e P. macrobotrys (Ruiz \& Pav.) Roem. \& Schult. As áreas de coleta no Distrito Federal foram: Parque Nacional de Brasília, Estação Ecológica de Águas Emendadas e Reseva Ecológica do IBGE. As espécies Palicourea marcgravii e P. officinalis apresentaram-se como distílicas em função da presença dos dois morfos florais, da sua razão equilibrada e do sistema de incompatibilidade auto e intramorfo. As espécies $P$. coriacea e $P$. macrobotrys apresentaram-se como variantes pela ocorrência de indivíduos com morfo homostilo, além da distilia na primeira espécie e pelo morfo homostilo ser dominante na segunda espécie. Não se sabe ainda as forças evolutivas que determinam essas variações, contudo acredita-se que as características genéticas intrínsecas de cada táxon têm uma considerável influência e que cada espécie pode responder de forma diferente, ou até mesmo não responder, perante as mesmas pressões seletivas a que estão submetidas.

Palavras-chave - biologia reprodutiva, heterostilia, isopletia, morfologia floral, polinização

\section{Introdução}

O gênero Palicourea tem uma riqueza de 200 espécies representadas por arbustos e arvoretas localizadas em diversas formações vegetacionais do Novo Mundo (Taylor 1997). O Cerrado possui aproximadamente 14 dessas

1. Parte da tese de doutorado do primeiro autor, Programa de Pós-Graduação em Ecologia, Universidade de Brasília, Brasília.

2. Universidade Federal de Goiás, Campus Catalão, Departamento de Ciências Biológicas, Setor Universitário, 75700-000 Catalão, GO, Brasil.

3. Universidade de Brasília, Instituto de Ciências Biológicas, Departamento de Ecologia, Asa Norte, 70910-900 Brasília, DF, Brasil.

4. Universidade Federal de Uberlândia, Instituto de Biologia, Caixa Postal 593, 38400-902 Uberlândia, MG, Brasil.

5._Autor para correspondência: helderconsolaro@gmail.com espécies distribuídas em suas variadas fitofisionomias (Mendonça et al. 2008) e, por essa razão, a região do Planalto Central é considerada como um dos centros de riqueza do gênero. Suas flores apresentam corola tubular, sem odor, de cores fortes e polinizadas principalmente por beija-flores (Taylor 1997).

Dentre as 25 famílias distílicas, Rubiaceae é considerada a mais rica, tendo, provavelmente, até mais espécies do que todas as outras famílias juntas (Ganders 1979, Barrett et al. 2000). Acredita-se que a distilia tenha surgido mais de uma vez na família, porém para Palicourea esta característica é considerada monofilética e comum em quase todas as espécies (Pailler \& Thompson 1997, Taylor 1997). Embora a distilia seja considerada primitiva e bem estabelecida na família e também no gênero, estudos encontraram 
diversas modificações evolutivas (Bawa \& Beach 1983, Sobrevila et al. 1983, Hamilton 1990, Richards \& Koptur 1993, Pailler et al. 1998, Faivre \& McDade 2001, Coelho \& Barbosa 2003, Castro et al. 2004, Consolaro et al. 2005). É relatado que a distilia pode originar a dioicia e o monomorfismo (Ganders 1979, Sobrevila et al. 1983, Hamilton 1990), contudo para Palicourea parece que a homostilia é a forma derivada mais comum. Morfologicamente, a homostilia caracteriza-se por suas flores apresentarem anteras e estigmas na mesma altura, sendo essa arquitetura evolutivamente derivada da distilia (Hamilton 1990). Em P. petiolaris (Sobrevila et al. 1983) algumas populações podem ser monomórficas, contudo o esperado se confirma nas populações homostílicas de P. alpina (Tanner 1982) e P. macrobotrys (Coelho \& Barbosa 2003); essa última, uma espécie típica de mata de galeria do Cerrado brasileiro.

Um trabalho teórico que utiliza programas computacionais capazes de gerenciar a entrada, a saída, a taxa de autofertilidade (s) e a depressão endogâmica $(\delta)$ de determinados morfos, demonstra que indivíduos com morfo homostilo com "s" maior do que zero e " $\delta$ " menor do que 0,5 , quando são inseridos em populações distílicas hipotéticas, tendem a aumentar sua freqüência e, gradualmente, ao longo das gerações, substituir os outros morfos. Juntamente a isso, o estudo também demonstra que quando o morfo inserido é homostilo longistilo, a substituição total do morfo tipicamente distílico e/ou do homostilo brevistilo é inevitável, ao passo que a dominância total do morfo homostilo brevistilo só é possível quando inserido na ausência do homostilo longistilo (ver detalhes em Charlesworth \& Charlesworth 1979).

Espécies ou populações que apresentam desvios em suas características distílicas são consideradas, na maioria das vezes, produtos da quebra e/ou permutação ("crossing over") do gene que controla a distilia (supergene) (Bawa \& Beach 1983, Hamilton 1990). A relação planta-polinizador desempenha um importante papel na manutenção do sistema distílico em função da dependência do fluxo do pólen intermorfo, ao passo que um desequilíbrio nessa interação pode exercer pressões seletivas a favor da quebra do supergene (Ganders 1979). Diversos fatores podem afetar a relação planta-polinizador, como perturbações ambientais (Barrett 1988), tamanho populacional reduzido (Kéry et al. 2000), reprodução clonal (Barrett 1977) e isolamento geográfico (Sobrevila et al. 1983, Washitani et al. 1994).

O presente estudo tem como finalidade investigar aspectos relacionados ao sistema distílico, como morfologia floral, isopletia, sistema de incompatibilidade e biologia da polinização de quatro espécies de Palicourea distribuídas no Cerrado do Brasil Central.

\section{Material e métodos}

Área de estudo - O presente estudo foi desenvolvido de agosto de 2005 a janeiro de 2007 em três áreas pertencentes à Reserva da Biosfera do Cerrado, Distrito Federal, Brasil: Parque Nacional de Brasília (Parna/Brasília) com uma área de 30.000 ha situada a 10 km da região central de Brasília; Estação Ecológica de Águas Emendadas (Esecae) com cerca de 10.500 ha localizada a 50 km de Brasília; Estação Ecológica do IBGE (Recor) com, aproximadamente, 1.360 ha situada a $35 \mathrm{~km}$ ao sul de Brasília. O clima das áreas é do tipo CW (classificação de Köppen) com estação seca de maio a setembro e chuvosa de outubro a abril, temperatura média de $21^{\circ} \mathrm{C}$, precipitação média de $1.550 \mathrm{~mm}$ e altitude de 1.000 a $1.150 \mathrm{~m}$.

Espécies estudadas - Foram estudadas quatro espécies de Palicourea: duas de hábito sub-arbustivo distribuídas nos campos limpos e sujos do bioma, P. coriacea (Cham.) K. Schum. e P. officinalis Mart., e outras duas arbustivas de matas de galeria, P. macrobotrys (Ruiz \& Pav.) Roem. \& Schult. e P. marcgravii A. St.-Hil. Para cada espécie, foi estudada uma população, sendo a de $P$. coriacea localizada na Recor, a de $P$. officinalis no Parna e a de P. macrobotrys e P. marcgravii na Esecae. As flores dessas espécies possuem corola tubular, antese nas primeiras horas da manhã, longevidade floral de aproximadamente 12 horas, receptividade estigmática e liberação polínica logo após o início da antese e o néctar é o principal recurso floral (Consolaro 2008).

Os espécimes testemunhos foram depositados no Herbário da Universidade de Brasília (UB 38156 e 38164) e no Herbário da Reserva Ecológica do IBGE (IBGE 64301 a 64303, 64306 a 64309 e 64310 a 64312).

Morfometria floral e razão dos morfos - A morfometria foi realizada com o auxílio de um paquímetro digital (erro 0,01 mm) e um estereomicroscópio Carl Zeiss DL 0195. As flores foram coletadas em diferentes indivíduos de forma aleatória e, posteriormente, armazenadas em etanol 70\%. O número de flores por morfo variou de acordo com as situações populacionais encontradas no campo. As medidas retiradas foram: comprimento do estigma (CE), comprimento do estilete (CEs), comprimento do tubo da corola (CC), comprimento do filete (CF), comprimento da antera (CA), altura da antera $(\mathrm{AA}=\mathrm{CA}+\mathrm{CF})$, altura do estigma $(\mathrm{AE}=\mathrm{CE}+\mathrm{CEs}) \mathrm{e}$ separação estigma-antera ( $\mathrm{AE}$ - AA). Correspondências nas alturas dos verticilos masculino e feminino entre os morfos foram usadas como indicador de presença de hercogamia recíproca. As comparações morfométricas entre os morfos de cada espécie foram obtidas por meio da ANOVA para dois fatores (morfo e verticilo reprodutivo). Quando os dados não alcançaram normalidade, o teste foi realizado com 
os valores ranqueados. O teste Tukey (HSD) foi utilizado para comparações múltiplas a posteriori após interação significativa entre os fatores. Coeficientes de Correlação de Spearman foram calculados para espécies que apresentaram diferenças no tamanho da corola. Todas as análises acima descriminadas foram realizadas utilizando o Programa Statistica versão 6.0 (StatSoft, Inc. 2001). Como a espécie $P$. coriacea apresentou um morfo variante juntamente com os morfos distílicos, a análise MANOVA foi utilizada para averiguar o efeito desse morfo na morfotipagem. Posteriormente, foi realizada Análise de Discriminantes (ADS) para constatar o grau de confiabilidade dessa separação (Versão R).

Para $P$. coriacea que, possivelmente, apresentava desvios na manifestação dos morfos distílicos, foram analisadas exsicatas em herbários com o intuito de verificar a posição das anteras e dos estigmas em flores de outras populações. Quando não foi possível a verificação direta dos verticilos nas exsicatas, flores de duplicatas foram retiradas e, posteriormente em laboratório, hidratadas, analisadas e morfotipadas. Os herbários visitados foram: Universidade de Brasília (UB), Universidade Federal de Mato Grosso do Sul (CGMS), Universidade Federal de Mato Grosso (UFMT), Universidade Federal de Goiás (UFG), Universidade Federal de Minas Gerais (BHCB) e Universidade de Campinas (UEC). O mesmo não foi feito para $P$. macrobotrys por tal análise ter sido realizada por Coelho \& Barbosa (2003).

A partir das condições morfológicas encontradas nas populações das quatro espécies estudadas, foi elaborado um fluxograma evolutivo para o gênero baseado nos resultados encontrados no modelo criado por Charlesworth \& Charlesworth (1979).

Para averiguar a ocorrência de isopletia na área de estudo, foram morfotipados e levantados ao acaso os indivíduos que ocorriam ao longo de um transecto de $100 \mathrm{~m}$. Foi realizado o teste de Qui-quadrado para testar a razão isoplética dos morfos florais na população $(P<0,05)$.

Sistema reprodutivo - O sistema de incompatibilidade foi determinado a partir da taxa de frutificação quantificada após experimentos de polinização manual e também a partir de observações do crescimento dos tubos polínicos. Os tipos de tratamentos de polinização seguiram o protocolo adotado em estudos de sistema reprodutivo de espécies distílicas (Bawa \& Beach 1983). Para as espécies que apresentaram o morfo homostilo, tal protocolo foi adaptado com seus botões sendo emasculados antes da antese para aumentar a confiabilidade do tratamento proposto. Vale ressaltar que as polinizações manuais foram sempre realizadas entre $08 \mathrm{~h} 00$ e $16 \mathrm{~h} 00$. As observações do crescimento dos tubos polínicos foram feitas usando a técnica de fluorescência (Martin 1959) a partir dos tratamentos de autopolinização, polinização intermorfo e intramorfo; os pistilos foram fixados após quatro, sete e 10 horas após estes tratamentos. Foi utilizado no mínimo 10 indivíduos por morfo e sete pistilos por tratamento por intervalo de tempo.
Visitantes florais e produção de néctar - Os visitantes florais foram determinados por observação direta das $06 \mathrm{~h} 00$ às $18 \mathrm{~h} 00$ em diferentes dias. O tempo total de observação por espécie foi de 15 horas para Palicourea officinalis e P. macrobotrys, 30 horas para $P$. marcgravii e 32 horas para $P$. coriacea. Os polinizadores principais foram determinados de acordo com a morfologia e comportamento dos visitantes, estrutura floral e freqüência de visitas. A identificação das aves foi feita por meio de documentação fotográfica e observação direta no campo, e dos insetos por meio de coletas e posterior envio a especialistas.

O volume total, concentração de açúcar e quantidade total de açúcar no néctar foram verificados separadamente para os morfos das quatro espécies em flores ensacadas em pré-antese. O volume foi medido com auxílio de capilares micrometrados, a concentração com refratômetro de mão e a quantidade de açúcar pelo protocolo descrito em Galetto \& Bernardello (2005). As medidas foram coletadas ao final do dia, antes do fim da longevidade. As análises estatísticas foram feitas com o teste Mann-Whitney $(U)$ para espécies com dois morfos e teste de Kruskal-Wallis $(H)$ para espécies com três morfos.

\section{Resultados}

Morfometria floral e razão dos morfos - Dentre as quatro espécies estudadas de Palicourea, duas podem ser consideradas como distílicas, pois os morfos brevistilo e longistilo estavam presentes na população e a isopletia foi constatada (P. officinalis e P. marcgravii) (tabela 1, figuras 1-4, 9A, B). A hercogamia recíproca não foi encontrada e o comprimento da corola e a separação estigma-antera foram significativamente maiores no morfo brevistilo do que no longistilo em ambas as espécies (tabela 1). As duas outras espécies do presente estudo, sendo uma espécie de formação campestre e outra de mata (P. coriacea e P. macrobotrys, respectivamente), não podem ser consideradas como distílicas, pois apresentaram variação na manifestação dos morfos (tabela 1, figuras 5-8, 9C, D). Foi elaborado um fluxograma evolutivo para o gênero Palicourea a partir das condições morfológicas encontradas nas quatro espécies estudadas (figura 10).

A população de $P$. coriacea apresentou o morfo longistilo e brevistilo, juntamente com um morfo homostilo (figuras 5-7, 9C), este último o mais freqüente na população (tabela 1). Entre as exsicatas analisadas $(n=30)$, foi observado flores das três morfologias. Vale ressaltar que todas as flores definidas como homostilas foram analisadas em laboratório. As exsicatas com flores homostilas foram provenientes de populações dos Estados do Mato Grosso do Sul, Mato Grosso, Minas Gerais e Goiás, sendo algumas delas coletadas 


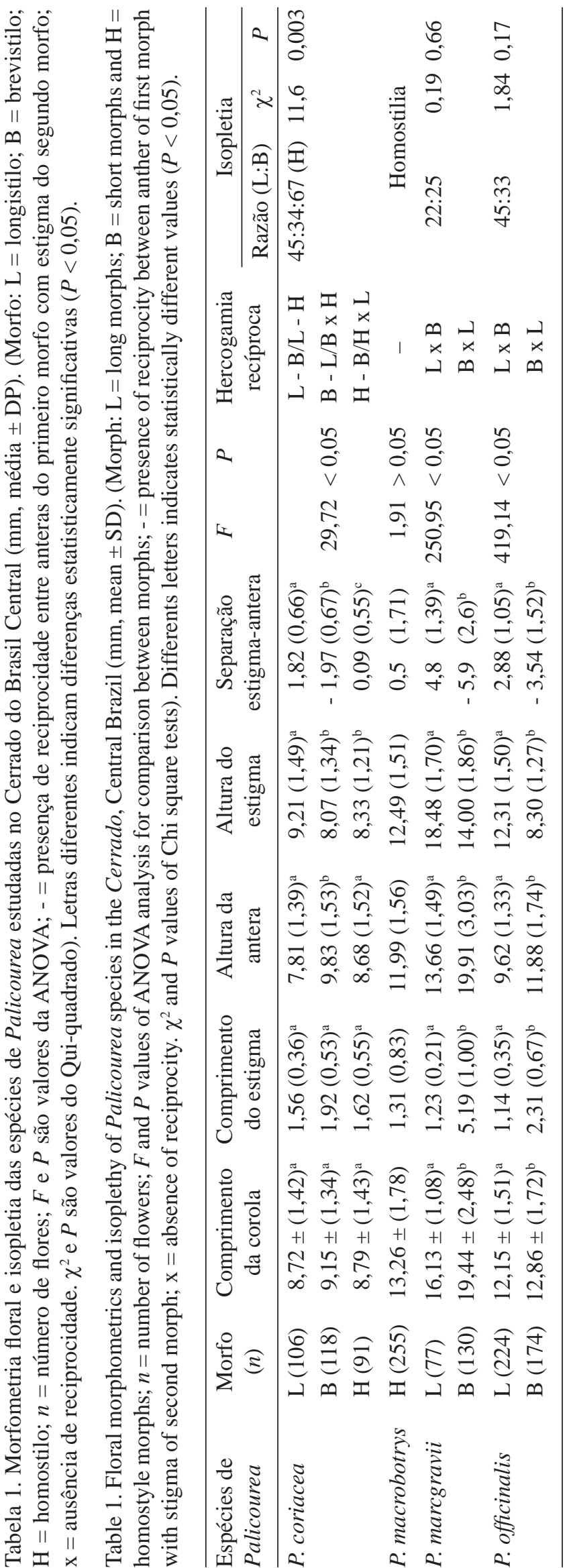

no mesmo município onde havia coletas anteriores com flores brevistilas e longistilas. A hercogamia recíproca foi encontrada entre os morfos distílicos e também entre as anteras do morfo longistilo com o estigma do homostilo e entre as anteras do homostilo com o estigma do brevistilo. Juntamente a essa combinação, a igualdade significativa da altura das anteras entre o morfo longistilo e o homostilo com o mesmo ocorrendo entre a altura do estigma do morfo brevistilo com o homostilo, indicam que, possivelmente, a forma homostila encontrada está mais próxima morfologicamente do morfo brevistilo (tabela 1, figura 7). A separação estigma-antera foi o único parâmetro em que o morfo brevistilo apresentou maior tamanho que o longistilo (tabela 1). A MANOVA indicou que existe diferença entre os três morfos $\left(F_{6,128}=47,003 ; P<0,05\right.$; Wilk's $\left.=0,097\right)$ e a ADS mostrou que a separação entre os morfos possui cerca de $97 \%$ de fidelidade.

As análises estatísticas realizadas para as flores mensuradas de Palicourea macrobotrys não demonstraram diferenças significativas entre elas, caracterizando a homostilia (tabela 1, figuras 8, 9D). Porém, é possível notar que suas flores apresentam características do morfo longistilo (homostilo longistilo); como tamanho dos lóbulos estigmáticos, localização do estigma próximo à entrada da corola e uma discreta disposição do estigma acima do nível das anteras (tabela 1, figuras 8, 9D).

Sistema reprodutivo - Os resultados dos tratamentos de polinização controlada de Palicourea marcgravii e $P$. officinalis foram semelhantes. As populações dessas espécies produziram significativamente mais frutos por meio de polinizações intermorfos do que por autopolinizações e polinizações intramorfos. Esses resultados indicam a condição de auto e intramorfo incompatibilidade em suas populações (tabela 2, figura 10). A frutificação após autopolinização, polinização intermorfo e intramorfo foi semelhante entre os morfos florais. O tratamento controle não apresentou diferença entre os morfos florais, sendo sua frutificação semelhante à polinização intermorfo e significativamente mais alta do que polinização auto e intramorfo (tabela 2).

As observações do crescimento do tubo polínico para $P$. marcgravii e $P$. officinalis também mostraram semelhanças. Após oito horas do tratamento já foi possível notar tubos no ovário, porém não foi observado penetração nos óvulos, possivelmente, pela sobreposição dos tecidos ovarianos. Os tubos gerados por polinizações intermorfos cresceram sem qualquer tipo de interrupção e, aparentemente, apresentaram-se 

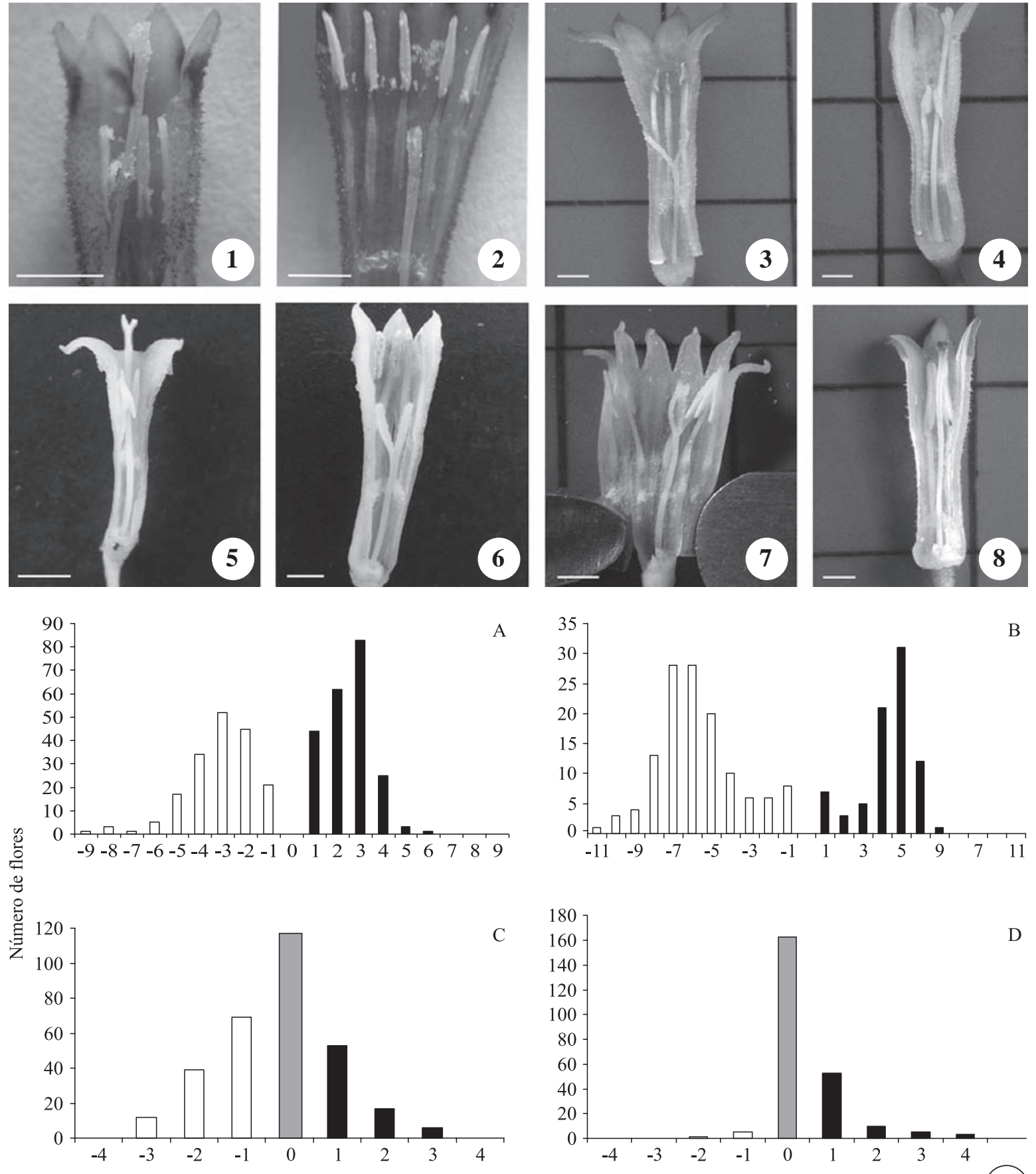

Separação estigma-antera (mm)

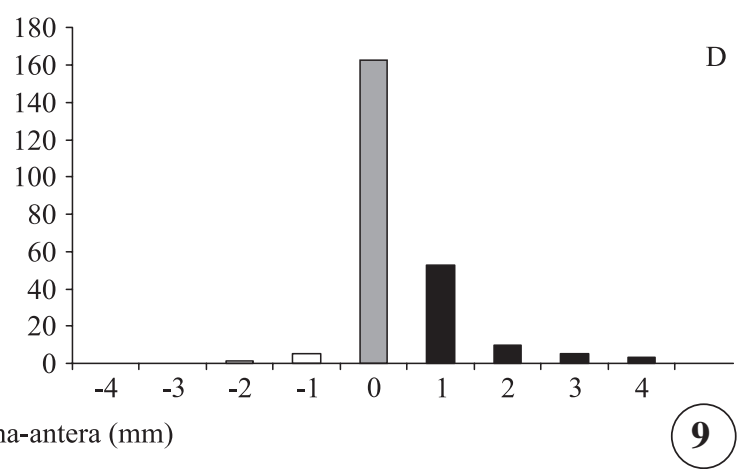

Figuras 1-9. Flores e representação gráfica dos morfos florais das espécies de Palicourea estudadas no Cerrado do Brasil Central. 1-2. Flor longistila e brevistila de P. officinalis, respectivamente. 3-4. Flor brevistila e longistila de P. marcgravii, respectivamente. 5-7. Flor longistila, brevistila e homostila de P. coriacea, respectivamente. 8. Flor homostila de P. macrobotrys. Barra $=2$ mm. 9. (A-D). Representação gráfica dos morfos florais. A. P. officinalis. B. P. marcgravii. C. P. coriacea. D. P. macrobotrys ( $\square$ = morfo brevistilo; $\mathbf{\square}$ = morfo longistilo; $\square=$ morfo homostilo).

Figures 1-9. Flowers and graphic representation of floral morphs of Palicourea species in the Cerrado, Federal District, Brazil. 1-2. Long and short flower of P. officinalis, respectively. 3-4. Short and long of P. marcgravii, respectively. 5-7. Long, short and homostyle flower of $P$. coriacea, respectively. 8. Homostyle flower of $P$. macrobotrys. Bar $=2 \mathrm{~mm}$. 9. (A-D). Graphic representation of floral morphs. A. P. officinalis. B. P. marcgravii. C. P. coriacea. D. P. macrobotrys $(\square=$ short morph; - = long morph; $\square=$ homostyle morph). 


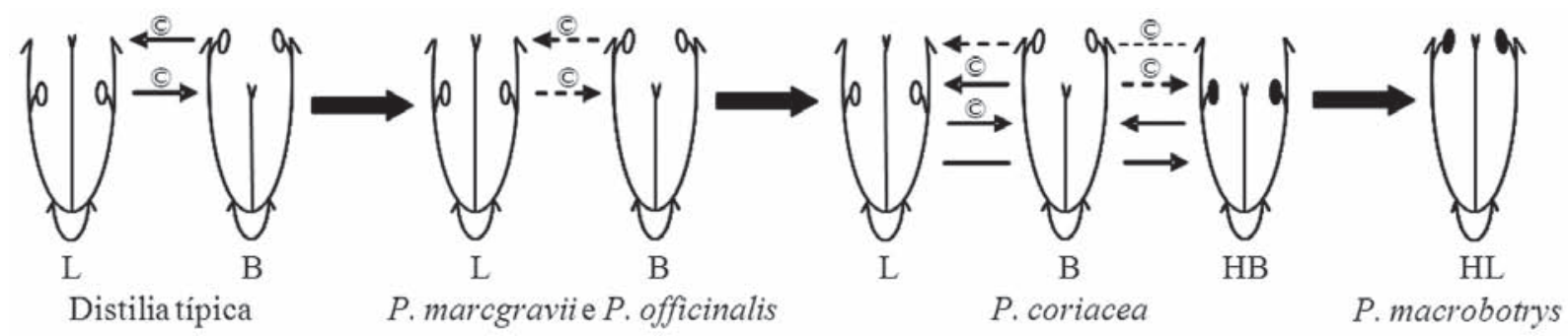

Figura 10. Fluxograma evolutivo, representação da hercogamia recíproca e relações de incompatibilidade das espécies de Palicourea estudadas no Cerrado do Brasil Central. ( $\mathrm{L}=$ morfo longistilo; $\mathrm{B}$ = brevistilo; $\mathrm{HB}$ = homostilo brevistilo; HL = homostilo longistilo; -- = ausência de hercogamia recíproca; - = presença de hercogamia recíproca; anteras pretas = autocompatibilidade; anteras brancas = auto e intramorfo incompatibilidade; $\mathbb{C}$ = intermorfo compatibilidade).

Figure 10. Evolutive diagram, reciprocal hercogamy representation and relation of incompatibility of Palicourea species in the Cerrado, Federal District, Brazil. (L = long morph; B = short morph; HB = short homostyle; HL = long homostyle; -- = ausence of reciprocal herkogamy; - = presence of reciprocal herkogamy; black anthers = self-compatibility; white anthers = self and intramorph incompatibility; $(\mathrm{C}=$ intermorph compatibility).

consistentes (maior diâmetro e mais fluorescência que os tubos polínicos de polinizações incompatíveis). A única diferença notada no sistema reprodutivo das duas espécies foi em relação ao comportamento dos tubos polínicos. No morfo brevistilo de P. marcgravii a interrupção do tubo polínico ocorreu no estigma após polinização auto e intramorfo, enquanto no morfo longistilo ocorreu ao longo do estilete. Em Palicourea officinalis, a interrupção ao longo do estilete foi observada nos dois morfos.

A população estudada de $P$. macrobotrys apresentou a condição de autocompatibilidade, formando frutos

Tabela 2. Resultados dos experimentos de polinização manual [\% de frutificação (número de flores)] das espécies de Palicourea estudadas no Cerrado do Brasil Central. (Morfo: $\mathrm{L}=$ longistilo; $\mathrm{B}=$ brevistilo; $\mathrm{H}=$ homostilo; $n=$ número de indivíduos. Experimentos: PInter = polinização intermorfo; PIntra = polinização intramorfo; $\mathrm{AP}=$ autopolinização; $\mathrm{Co}=$ controle). Dentro de cada espécie, a primeira letra representa comparações entre morfos e as letras conseguintes comparações entre experimentos. Letras diferentes indicam diferenças estatisticamente significativas $(P<0,05)$. Em PInter para $P$. coriacea: p. ex. BxL, o primeiro morfo representa o doador de pólen.

Table 2. Results of hand pollination experiments [\% of fruits (number of flowers)] of Palicourea species in the Cerrado, Federal District, Brazil. (Morph: $\mathrm{L}=$ long morph; $\mathrm{B}=$ short morph; $\mathrm{H}=$ homostyle morph; $n=$ number of individuals. Experiments: PInter = intermorph pollination; PIntra $=$ intramorph pollination; $\mathrm{AP}=$ self-pollination; $\mathrm{Co}=$ control). For each species, the first letter represents comparisons between morph, the others letters comparisons between experiments. Differents letters indicates statistically different values $(P<0,05)$. In PInter for $P$. coriacea: for example BxL, the first morph represents the pollen giver.

\begin{tabular}{|c|c|c|c|c|c|}
\hline \multirow{2}{*}{$\begin{array}{l}\text { Espécies de } \\
\text { Palicourea }\end{array}$} & \multirow{2}{*}{$\begin{array}{l}\text { Morfo } \\
(n)\end{array}$} & \multicolumn{4}{|c|}{ Experimentos } \\
\hline & & PInter & PIntra & $\mathrm{AP}$ & Co \\
\hline \multirow[t]{2}{*}{ P. marcgravii } & $\mathrm{L}(15)$ & $64^{\mathrm{a}, \mathrm{b}}(44)$ & $11^{\mathrm{h}, \mathrm{j}}(28)$ & $6^{\mathrm{d}, \mathrm{j}}(51)$ & $70^{\mathrm{c}, \mathrm{b}}(36)$ \\
\hline & B (15) & $78^{\mathrm{a}, \mathrm{b}}(41)$ & $2^{\mathrm{h}, \mathrm{i}}(56)$ & $2^{\mathrm{d}, \mathrm{i}}(48)$ & $57^{\mathrm{c}, \mathrm{b}}(35)$ \\
\hline \multirow[t]{2}{*}{ P. officinalis } & $\mathrm{L}(17)$ & $37^{\mathrm{a}, \mathrm{d}}(44)$ & $16^{\mathrm{l,n}}(42)$ & $5^{\mathrm{c,n}}(43)$ & $48^{\mathrm{d}}(40)$ \\
\hline & $\mathrm{B}(14)$ & $35^{\mathrm{a}, \mathrm{d}}(41)$ & $2^{1, \mathrm{~m}}(43)$ & $2^{\mathrm{c}, \mathrm{m}}(41)$ & $36^{d}(45)$ \\
\hline P. macrobotrys & H (26) & - & $76^{\mathrm{a}}(112)$ & $67^{\mathrm{a}}(115)$ & $75^{\mathrm{a}}(100)$ \\
\hline \multirow[t]{3}{*}{ P. coriacea } & L (15) & BxL: 36 (33)/HxL: 25 & $4^{\mathrm{opp}, \mathrm{r}, \mathrm{s}}(28)$ & $5^{\mathrm{b}, \mathrm{d}, \mathrm{f,p}}(42)$ & $24^{\mathrm{a}, \mathrm{e}}(42)$ \\
\hline & $\mathrm{B}(11)$ & LxB: $38^{\mathrm{e}}(40) / \mathrm{HxB}: 2.4^{\mathrm{fg}, \mathrm{gu}}(42)$ & $4^{\mathrm{o}, \mathrm{q}, \mathrm{tu},}(45)$ & $3^{\mathrm{f}, \mathrm{q}}(36)$ & $22^{\mathrm{e}}(41)$ \\
\hline & H (33) & LxH: 5 $5^{\mathrm{a}, \mathrm{c}}(40) / \mathrm{BxH}: 23^{\mathrm{f}, \mathrm{g}, \mathrm{v}}(35)$ & $25^{\mathrm{a}, \mathrm{s}, \mathrm{t}, \mathrm{v}}(40)$ & $13^{\mathrm{b}, \mathrm{c}, \mathrm{f}}(46)$ & $23^{\mathrm{a}, \mathrm{e}}(69)$ \\
\hline
\end{tabular}


tanto por autopolinizações quanto por polinizações cruzadas (tabela 2, figura 10). Os tubos polínicos já se encontravam no ovário de forma consistente a partir das quatro horas após autopolinização e polinização cruzada, contudo não foi observado penetração nos óvulos, provavelmente pelo mesmo motivo estrutural acima mencionado.

A relação de incompatibilidade entre os morfos tipicamente distílicos de $P$. coriacea seguiu o padrão heterostílico (figura 10). A produção de frutos foi significativamente maior após polinizações intermorfos do que após polinizações auto e intramorfo em ambos os morfos florais. A frutificação de cada tratamento realizado foi semelhante entre os morfos. A formação de frutos pelo tratamento controle foi semelhante à polinização intermorfo nos dois morfos, contudo foi significativamente mais alta do que em polinização auto e intramorfo (tabela 2). O tubo polínico se encontrava no ovário após seis horas do tratamento, mas não foi observado penetração nos óvulos. As observações mostraram que em polinização auto e intramorfo a interrupção do tubo polínico no morfo brevistilo ocorreu no estigma, enquanto no longistilo ocorreu no início do estilete.

Os resultados dos experimentos de polinização controlada dos morfos distílicos de P. coriacea com o morfo homostilo se mostraram de difícil compreensão. Foi encontrado que o morfo homostilo é autocompatível e que seu grão de pólen é compatível com o morfo longistilo. Além disso, compatibilidade também ocorreu entre o morfo brevistilo e o homostilo, porém o longistilo foi incompatível com o homostilo. Algumas diferenças foram encontradas envolvendo polinizações cruzadas intermorfos e o tratamento controle. A frutificação do morfo homostilo gerada pelo pólen do longistilo foi significativamente menor que o controle, o mesmo ocorrendo na recepção do pólen do homostilo pelo morfo brevistilo. A formação de frutos do tratamento controle foi semelhante à frutificação do morfo longistilo produzida pela recepção do pólen do homostilo e semelhante à frutificação do homostilo gerada pelo pólen do brevistilo (tabela 2, figura 10). Em todos os tratamentos compatíveis, o crescimento do tubo polínico já se encontrava no ovário após seis horas do tratamento, mas não foi possível observar penetração nos óvulos. A interrupção do tubo polínico brevistilo no morfo homostilo ocorreu ao longo do estilete. As análises entre os morfos distílicos mostraram que em polinização auto e intramorfo a interrupção do tubo polínico no morfo brevistilo ocorreu no estigma; enquanto no longistilo ocorreu ao longo do estilete.
Visitantes florais e produção de néctar - Beija-flores visitaram as flores de todas as espécies estudadas, independentemente da fitofisionomia, contudo apenas em P. marcgravii, P. macrobotrys e P. officinalis eles foram considerados como polinizadores principais. Para essas espécies, visitas esporádicas de borboletas também foram registradas, porém comportando-se como polinizadores ocasionais. Abelhas foram observadas apenas em $P$. coriacea, sendo elas e o beija-flor Heliactin cornuta considerados os principais visitantes. Juntamente a esse grupo, também foram observadas visitas de Diptera e Lepidoptera (tabela 3).

O volume total, concentração de açúcar e quantidade total de açúcar no néctar não diferiram significativamente entre os morfos para as quatro espécies estudadas (tabela 4).

\section{Discussão}

Por mais que alguns trabalhos discutam que a hercogamia recíproca é necessária para considerar uma espécie ou população verdadeiramente distílica (Pailler \& Thompson 1997, Faivre \& McDade 2001), acreditase que a distilia pode ser atribuída às populações de Palicourea marcgravii e P. officinalis pela presença dos dois morfos florais, pelo sistema de incompatibilidade, pelos dados de tubos polínicos e pela isopletia. A mesma atribuição não pode ser feita a $P$. coriacea, pois os morfos distílicos ocorreram juntamente com o homostilo, e a $P$. macrobotrys, pois o morfo homostilo ocorreu de forma exclusiva em sua população.

A homostilia se origina em muitos táxons distílicos de Rubiaceae, podendo manifestar-se como caráter exclusivo em uma população ou, mais frequentemente, em apenas alguns indivíduos dentro de uma população distílica (Ganders 1979, Hamilton 1990, Coelho \& Barbosa 2003). Acredita-se que cada espécie ou população pode responder de forma diferente perante as mesmas pressões seletivas a que estão submetidas (Charlesworth \& Charlesworth 1979, Li \& Johnston 2001), contudo parece que nas espécies de Palicourea a homostilia é o caminho evolutivo mais freqüente, como encontrado em P. alpina (Sw.) DC. (Tanner 1982), P. macrobotrys (Ruiz \& Pav.) Roem. \& Schult. (Coelho \& Barbosa 2003) e, possivelmente, em $P$. grandifolia (Willd. ex Roem. \& Schult.) Standl., P. guianensis Aubl. e P. nitidella (Müll. Arg.) Standl. (Consolaro 2008). Outros casos de homostilia originada de espécies distílicas de Rubiaceae também foram registrados, como Pentas lanceolata (Forssk.) K. Schum., P. zanzibarica (Klotzsch) Vatke, P. parviflora Benth., Adenosacme longifolia (Wall.) Hook. 
Tabela 3. Visitantes florais, número total de visitas, freqüência e categoria dos visitantes de quatro espécies de Palicourea estudadas no Cerrado do Brasil Central. (MF = muito freqüente ( $>5$ visitas/hora); $\mathrm{F}=$ frequente $( \pm 1$ visita/hora); $\mathrm{R}=$ raro ( $<1$ visita/hora); $\mathrm{PP}$ = polinizador principal; $\mathrm{PO}=$ polinizador ocasional $)$.

Table 3. Floral visitors, total number of visits, frequency and category of visitors of Palicourea species in the Cerrado, Federal District, Brazil. $(\mathrm{MF}=$ very frequent $(>5$ visits/hour $) ; \mathrm{F}=$ frequent $( \pm 1$ visit/hour $) ; \mathrm{R}=$ rare $(<1$ visit/hour $) ; \mathrm{PP}=$ main pollinator; $\mathrm{PO}=$ occasional pollinator).

\begin{tabular}{|c|c|c|c|c|}
\hline $\begin{array}{l}\text { Espécies de } \\
\text { Palicourea }\end{array}$ & Visitantes florais & Visitas & Frequência & Categoria \\
\hline \multirow[t]{7}{*}{ P. officinalis } & Apodiformes & & & \\
\hline & Amazilia fimbriata (Gmelin 1788) & 26 & $\mathrm{~F}$ & $\mathrm{PP}$ \\
\hline & Chlorostilbon aureoventris (d’Orbigny \& Lafresnaye 1838) & 25 & $\mathrm{~F}$ & PP \\
\hline & Heliactin cornuta (Wied 1821) & 2 & $\mathrm{R}$ & $\mathrm{PO}$ \\
\hline & Lepidoptera & & & \\
\hline & Phoelis philea (Johansson 1767) & 11 & $\mathrm{R}$ & $\mathrm{PO}$ \\
\hline & Xeniades chalestra (Hewitson 1866) & 1 & $\mathrm{R}$ & $\mathrm{PO}$ \\
\hline \multirow[t]{6}{*}{ P. marcgravii } & Apodiformes & & & \\
\hline & Amazilia fimbriata (Gmelin 1788) & 35 & MF & $\mathrm{PP}$ \\
\hline & Chlorostilbon sp. & 3 & $\mathrm{R}$ & $\mathrm{PO}$ \\
\hline & Lepidoptera & & & \\
\hline & Heliconius erato phyllis (Fabricius 1775) & 3 & $\mathrm{R}$ & $\mathrm{PO}$ \\
\hline & H. ethilla macaea (Godan 1819) & 3 & $\mathrm{R}$ & $\mathrm{PO}$ \\
\hline \multirow[t]{4}{*}{ P. macrobotrys } & Apodiformes & & & \\
\hline & Thalurania furcata (Gmelin 1788) & 152 & MF & $\mathrm{PP}$ \\
\hline & Lepidoptera & & & \\
\hline & Hypothyris ninonia (Boisduval 1836) & 5 & $\mathrm{R}$ & $\mathrm{PO}$ \\
\hline \multirow[t]{19}{*}{ P. coriacea } & Apodiformes & & & \\
\hline & Heliactin cornuta (Wied 1821) & 60 & $\mathrm{~F}$ & PP \\
\hline & Apoidea & & & \\
\hline & Bombus atratus Frankin 1913 & 80 & $\mathrm{~F}$ & PP \\
\hline & Euglossa melanotricha Moure 1967 & 20 & F & PP \\
\hline & Ceratina sp. & 28 & $\mathrm{~F}$ & PP \\
\hline & Oxaea flavescens Klug 1807 & 6 & $\mathrm{R}$ & $\mathrm{PO}$ \\
\hline & Megachile sp. & 2 & $\mathrm{R}$ & $\mathrm{PO}$ \\
\hline & Epicharis bicolor Smith 1854 & 2 & $\mathrm{R}$ & $\mathrm{PO}$ \\
\hline & Halictidae & & & \\
\hline & Morfo-espécie 1 & 20 & $\mathrm{~F}$ & PP \\
\hline & Morfo-espécie 2 & 20 & $\mathrm{~F}$ & PP \\
\hline & Morfo-espécie 3 & 25 & F & PP \\
\hline & Morfo-espécie 4 & 10 & $\mathrm{R}$ & $\mathrm{PO}$ \\
\hline & Lepidoptera & & & \\
\hline & Stalachtis phlegia (Cramer 1775) & 2 & $\mathrm{R}$ & $\mathrm{PO}$ \\
\hline & Lerema veadeira (Mielke 1968) & 4 & $\mathrm{R}$ & $\mathrm{PO}$ \\
\hline & Diptera & & & \\
\hline & Morfo-espécie 1 & 2 & $\mathrm{R}$ & $\mathrm{PO}$ \\
\hline
\end{tabular}


Tabela 4. Volume, concentração e quantidade total de açúcar no néctar das espécies de Palicourea estudadas no Cerrado do Brasil Central. (Morfo: L = longistilo; B = brevistilo; $\mathrm{H}=$ homostilo; $n$ = número de flores).

Table 4. Volume, concentration and total amount of sugar in the nectar of Palicourea species in the Cerrado, Federal District, Brazil. (Morph: $\mathrm{L}=$ long; $\mathrm{B}=$ short; $\mathrm{H}$ = homostyle; $n$ = number of flowers).

\begin{tabular}{lcccc}
\hline $\begin{array}{l}\text { Espécies de } \\
\text { Palicourea }\end{array}$ & $\begin{array}{c}\text { Morfo } \\
(n)\end{array}$ & $\begin{array}{c}\text { Volume } \\
(\mu \mathrm{L})\end{array}$ & $\begin{array}{c}\text { Concentração } \\
(\%)\end{array}$ & $\begin{array}{c}\text { Quantidade } \\
(\mathrm{mg})\end{array}$ \\
\hline P. coriacea & L (15) & $2,02 \pm 0,94$ & $25,60 \pm 2,50$ & $0,57 \pm 0,28$ \\
& B (5) & $1,93 \pm 0,55$ & $21,40 \pm 4,10$ & $0,42 \pm 0,06$ \\
P. macrobotrys & H (33) & $1,92 \pm 0,93$ & $27,00 \pm 2,60$ & $0,56 \pm 0,23$ \\
P. marcgravii & H (10) & $4,99 \pm 3,17$ & $17,90 \pm 2,18$ & $0,89 \pm 0,42$ \\
& L (15) & $5,83 \pm 3,07$ & $17,47 \pm 2,35$ & $1,03 \pm 0,44$ \\
P. officinalis & B (15) & $5,83 \pm 2,96$ & $16,46 \pm 2,06$ & $0,98 \pm 0,43$ \\
& L (32) & $3,39 \pm 1,59$ & $14,76 \pm 5,50$ & $0,49 \pm 0,26$ \\
& B (30) & $3,64 \pm 1,31$ & $14,63 \pm 5,56$ & $0,63 \pm 0,41$ \\
\hline
\end{tabular}

f., Pleiocraterium verticillare (Wall. ex Wight \& Arn.) Bremek., Parapentas setigera (Hiern) Verdc., Hedyotis subviscosa (Wright ex A. Gray) Shinners, Oldenlandia herbacea (L.) Roxb., O. umbellata L. (Bir Bahadur 1968, em tabela com dados compilados de outros autores), Mitchella repens L. (Ganders 1975) e algumas espécies de Psychotria (Hamilton 1990).

Embora o modelo de Charlesworth \& Charlesworth (1979) tenha sido realizado ao nível populacional e o fluxograma evolutivo de Palicourea pela junção das formas de homostilia presentes no gênero, o uso desse modelo como referência é aceitável, pois a "substituição gradual” e as "espécies”, na verdade, representam estágios de um processo com causas finais, até certo ponto semelhantes, ou seja, a dominância da homostilia. Com base nesse modelo, suspeita-se que $P$. coriacea possa estar passando pelo mesmo processo de substituição gradual dos morfos observados no trabalho desses autores, já que o morfo homostilo é autocompatível e os morfos distílicos são auto e intramorfo incompatíveis. Paralelamente a isso, a ocorrência integral da homostilia em P. macrobotrys, aliada com a mesma constatação feita em populações da região do Triângulo Mineiro (Coelho \& Barbosa 2003), pode sugerir que essa espécie tenha passado pela fase evolutiva que $P$. coriacea se encontra e que a quebra da distilia para homostilia pode ocorrer independentemente em várias espécies ou populações. Levando em consideração as situações encontradas em $P$. coriacea e $P$. macrobotrys, juntamente com a ausência de hercogamia recíproca em $P$. marcgravii e $P$. officinalis, é possível dizer que cada uma dessas quatro espécies esteja em um estágio diferente no curso evolutivo da homostilia e a não reciprocidade nessas duas espécies representaria o primeiro passo para a quebra da distilia. Alguns trabalhos demonstram que mesmo que o sistema de incompatibilidade esteja funcionando, como encontrado nas espécies em questão, se o fluxo de pólen estiver em desequilíbrio, talvez pela ausência de hercogamia recíproca, a quebra do supergene distílico pode ocorrer (Endels et al. 2002). Foi proposto um caminho evolutivo para a manifestação da homostilia dentro do gênero, porém vale ressaltar que tal fluxograma seria apenas uma ideia e uma maneira de incentivar estudos mais detalhados a respeito do tema.

A hercogamia recíproca é tida como o principal fator que intermedeia a troca de pólen intermorfo via interação com os polinizadores, de maneira que imprecisão na reciprocidade e conseqüente desequilíbrio nessa interação podem explicar variações na expressão de suas características morfológicas e quedas no sucesso reprodutivo do grupo (Ganders 1979, Faivre \& McDade 2001). Entretanto, de acordo com os resultados de biologia da polinização, produção de néctar e sistema reprodutivo, esse desequilíbrio ainda não se manifestou em $P$. marcgravii e $P$. officinalis, uma vez que foram constatados agentes polinizadores eficientes, nenhuma oferta diferenciada de néctar por parte dos morfos e sucesso reprodutivo similar entre as flores longistilas e brevistilas.

A ornitofilia é considerada como dominante em Palicourea (Taylor 1997), sendo confirmada em vários trabalhos que abordam a biologia da polinização no 
gênero (Sobrevila et al. 1983, Ree 1997, Contreras \& Ornelas 1999, Coelho \& Barbosa 2003), incluindo o presente estudo. Palicourea coriacea foi a única espécie na qual as abelhas também foram consideradas como polinizadores principais, uma condição rara para o gênero. Em um estudo com P. padifolia, as abelhas também foram os principais visitantes, entretanto a sua eficiência no fluxo de pólen intermorfo foi questionada e discutido seu papel na quebra da distilia (Ree 1997). Como a ornitofilia é considerada predominante no gênero e a melitofilia foi a principal síndrome de uma espécie com variação nos seus padrões distílicos (P. coriacea), é de certa forma coerente pensar que essa mudança no tipo de polinizador pode ter influenciado de alguma maneira a evolução de indivíduos homostílicos na população. Seguindo a mesma linha de discussão, a homostilia em $P$. macrobotrys refutaria tal questionamento, já que as observações dos visitantes florais confirmaram a síndrome mais comum do gênero com os polinizadores, aparentemente, desempenhando de maneira eficiente sua função. Um contexto como esse, onde duas espécies apresentam o morfo variante homostilo, mas apenas uma manifesta mudança nos visitantes florais, pode demonstrar que algumas populações são mais vulneráveis que outras a alterações no serviço de polinização. Para algumas espécies, o papel dos polinizadores no fluxo de pólen intermorfo não parece ser tão importante quanto se espera e outras pressões seletivas, possivelmente, podem ser mais importantes que o desequilíbrio no fluxo de pólen na origem de variações na distilia.

Homostilia derivada da distilia por meio do "crossing over" do supergene distílico pode manifestar-se como: "homostilia longistila", estames na mesma altura do estigma na flor longistila; "homostilia brevistila", estames no mesmo nível do estigma na flor brevistila; e "homostilia intermediária”, estames e estigma no nível intermediário entre os verticilos reprodutivos dos morfos longistilos e brevistilos (Hamilton 1990). A combinação de reciprocidades encontrada entre o morfo homostilo e os morfos distílicos em $P$. coriacea, conjuntamente com as manifestações de incompatibilidade, sugerem que a homostilia encontrada na população realmente é do tipo "homostilia brevistila". Segundo o modelo de Charlesworth \& Charlesworth (1979), essa forma homostílica somente substitui o morfo longistilo e brevistilo na ausência de uma "homostilia longistila", situação que parece ocorrer na população estudada em função da homostilia brevistila ser exclusiva e significativamente mais freqüente que os outros dois morfos. Em P. macrobotrys a condição é diferente, pois suas características morfológicas indicam que a homostilia é do tipo "homostilia longistila". Os dois morfos homostilos encontrados em Palicourea sugerem que a origem da homostilia pode ocorrer de maneira independente num mesmo táxon e que as pressões seletivas locais somadas as características genéticas intrínsecas de cada espécie podem determinar o sentido do caminho evolutivo a ser seguido.

Morfo brevistilo com estruturas morfológicas maiores do que o longistilo, como encontrado na maioria das estruturas mensuradas, é comum em sistemas distílicos, na família Rubiaceae e em Palicourea (Ganders 1979, Sobrevila et al. 1983, Ree 1997, Contreras \& Ornelas 1999, Li \& Johnston 2001, Castro et al. 2004). Acredita-se que as estruturas morfológicas maiores no morfo brevistilo é uma maneira de compensar uma possível assimetria no fluxo de pólen em sua direção, devido a disposição estrutural do morfo longistilo facilitar a polinização (Ganders 1979). Realmente, alguns trabalhos têm demonstrado que o "display” floral exerce uma função importante em sistemas reprodutivos intermediado por animais, contudo estudos direcionados para espécies distílicas seriam necessários para constatar até que ponto estruturas morfológicas maiores entre os morfos influenciam na atração do polinizador (Galen \& Stanton 1989, Mal \& Lovett-Doust 1997). Além disso, outros estudos mostram que nem sempre essa diferença existe ou ocorre nesse sentido (Stone 1995, Teixeira \& Machado 2004, Coelho \& Barbosa 2004, Pereira et al. 2006).

Variações no comportamento reprodutivo entre espécies cogenéricas são frequentemente encontradas (Washitani et al. 1994, Castro et al. 2004), podendo, muitas vezes, manifestar-se na própria frutificação. A frutificação gerada a partir da polinização intermorfo de P. marcgravii, P. officinalis e P. coriacea foi semelhante, porém nota-se uma diferença na frutificação entre as espécies. Muitas espécies distílicas de Rubiaceae (Castro et al. 2004) apresentam frutificação similar a $P$. officinalis e $P$. coriacea, entretanto ela é considerada baixa quando comparada à frutificação encontrada nas populações estudadas de $P$. marcgravii e $P$. macrobotrys, em Palicourea fendleri Standl. (82,4\% brevistilo e 60\% longistilo) (Sobrevila et al. 1983), Psychotria jasminoides Standl. (70\% e 72,1\%) (Castro et al. 2004) e P. poepippigiana Mull. Arg. (76,2\% e 84,2\%) (Coelho $\&$ Barbosa 2004). Visto que a população de $P$. coriacea não é distílica, seria compreensível pensar que a sua menor frutificação seria uma conseqüência da variação morfológica apresentada, contudo, pela população de $P$. officinalis ter sido considerada tipicamente distílica e por elas terem frutificação parecida, tal suposição foi 
descartada. Como as populações foram estudadas em áreas separadas e aquelas que apresentaram frutificação diferente não ocorreram na mesma fitofisionomia, acredita-se que a variação encontrada entre elas pode estar associada a algum fator abiótico (Sutherland 1986).

Os variados comportamentos de incompatibilidade observados nas espécies estudadas contribuem para as incertezas quanto à origem e os mecanismos que atuam nesse processo. Como o sistema de incompatibilidade de espécies distílicas é do tipo esporofítico, esperava-se que a reação de incompatibilidade ocorresse na superfície estigmática, contudo os resultados encontrados, juntamente com estudos recentes, demostram que em plantas heterostílicas as regiões de interrupção do tubo polínico diferem entre os morfos e podem envolver o estigma, estilete ou ovário (Anderson \& Barrett 1986, Coelho \& Barbosa 2004, Pereira et al. 2006, Consolaro 2008). Estudos de incompatibilidade em espécies distílicas de Rubiaceae têm demonstrado claramente essas diferenças; o local de inibição é diferente tanto entre espécies quanto entre os morfos, sendo caracterizado principalmente pela interrupção ao longo do estilete no morfo longistilo e no estigma no brevistilo (Bawa \& Beach 1983, Passos \& Sazima 1995, Teixeira \& Machado 2004, Coelho \& Barbosa 2004, Pereira et al. 2006), como encontrado em Palicourea marcgravii e nos morfos distílicos de $P$. coriacea. Porém, para enfatizar a diversidade de mecanismo que pode ser encontrada nesses grupos, em $P$. officinalis e em um estudo com $P$. longepedunculata (Pereira et al. 2006) foi observado que a interrupção em ambos os morfos ocorreu ao longo do estilete.

Os resultados encontrados para as quatro espécies de Palicourea revelam situações evolutivas e ecológicas interessantes a respeito do tema, sendo possível fazer algumas considerações. A) Espécies inseridas dentro de um mesmo taxon, provavelmente, variam quanto a instabilidade genética e cada uma pode responder de forma diferente perante as mesmas alterações. B) A fitofisionomia, possivelmente, não exerce influência nos fatores que mantêm os padrões distílicos, pois tanto espécies de formações florestais quanto de formações campestres apresentaram populações tipicamente distílicas e populações variantes. C) A origem de variações evolutivas na distilia nem sempre está associada a mudanças no serviço de polinização. D) Quando o gênero Palicourea apresenta variações nos padrões distílicos no Cerrado do Brasil Central, elas são caracterizadas pela homostilia. Os resultados descritos no presente trabalho representam um dos primeiros passos no estudo da evolução e ecologia da distilia no bioma Cerrado, com isso várias linhas de pesquisas adicionais devem ser desenvolvidas para melhor entender e esclarecer as forças que regem o sistema, como: 1) Análises filogenéticas para confirmar ou refutar o fluxograma evolutivo sugerido para o gênero; 2) Estudos ecológicos direcionados para descobrir as pressões seletivas que agem na derivação da homostilia; 3) Estudos moleculares para entender os mecanismos que atuam na origem dos morfos homostilos.

Agradecimentos - À Dra. Solange Cristina Augusto, pela identificação das abelhas. Ao Dr. Piero Delprete, pela identificação das plantas. Ao Hélder A. Pereira, pela identificação dos Lepidópteros. Aos amigos Eddie Lenza e Felipe Rego, pelo auxílio nas análises estatísticas. Aos herbários (UB, CGMS, UFMT, UFG, BHCB e UEC), Parna, Esecae e Recor pela permissão para a coleta de dados. A Capes e CNPq, pela bolsa de doutorado referente ao primeiro autor.

\section{Referências bibliográficas}

ANDERSON, W.R. \& BARRETT, S.C.H. 1986. Pollen tube growth in tristylous Pontederia cordata (Pontederiaceae). Canadian Journal of Botany 64:2602-2607.

BARRETT, S.C.H. 1977. Tristyly in Eichhornia crassipes (Mart.) Solms. (water hyacinth). Biotropica 9:230-238.

BARRETT, S.C.H. 1988. The evolution, maintenance, and loss of self-incompatibility systems. In Plant reproductive ecology: patterns and strategies (L. Lovett Doust, ed.). University Press, New York. p.98-124.

BARRETT, S.C.H., WILKEN, D.H. \& COLE, W.W. 2000. Heterostyly in the Lamiaceae: the case of Salvia brandegeei. Plant Systematics and Evolution 223:211219.

BAWA, K.S. \& BEACH, J.H. 1983. Self-incompatibility systems in the Rubiaceae of a tropical lowland wet forest. American Journal of Botany 70:1281-1288.

BIR BAHADUR 1968. Heterostyly in Rubiaceae: a review. Journal of Osmania University (Science), Golden Jubilee Special Volume 1281-1288.

CASTRO, C.C., OLIVEIRA, P.E. \& ALVES, M.C. 2004. Breeding system and floral morphometry of distylous Psychotria L. species in the Atlantic Rain Forest, SE Brazil. Plant Biology 6:755-760.

CHARLESWORTH, D. \& CHARLESWORTH, B. 1979. A model for evolution of heterostyly. American Naturalist 114:467-498.

COELHO, C.P. \& BARBOSA, A.A. 2003. Biologia reprodutiva de Palicourea macrobotrys Ruiz \& Pavon (Rubiaceae): um possível caso de homostilia no gênero Palicourea Aubl. Revista Brasileira de Botânica 26: 403-413. 
COELHO, C.P. \& BARBOSA, A.A. 2004. Biologia reprodutiva de Psychotria poeppigiana Mull. Arg. (Rubiaceae) em mata de galeria. Acta Botanica Brasilica 18:481-489.

CONSOLARO, H., SILVA, E.B. \& OLIVEIRA, P.E.A.M. 2005. Variação floral e biologia reprodutiva de Manettia cordifolia Mart. (Rubiaceae). Revista Brasileira de Botânica 28:85-94.

CONSOLARO, H. 2008. A distilia em espécies de Rubiaceae do bioma Cerrado. Tese de doutorado, Universidade de Brasília, Brasília.

CONTRERAS, P.S. \& ORNELAS, J.F. 1999. Reproductive conflicts of Palicourea padifolia (Rubiaceae) a distylous shrub of a tropical cloud forest in Mexico. Plant Systematics and Evolution 219:225-241.

ENDELS, P., JACQUEMYN, H., BRYS, R. \& HERMY, M. 2002. Changes in pin-thrum ratios in populations of the heterostyle Primula vulgaris Huds: does imbalance affect populations persistence? Flora 197: 326-331.

FAIVRE, A.M. \& MCDADE, L.A. 2001. Population-level variation in the expression of heterostyly in three species of Rubiaceae: does reciprocal placement of anthers and stigmas characterize heterostyly? American Journal of Botany 88:841-853.

GALEN, C. \& STANTON, M.L. 1989. Bumble bee pollination and floral morphology: factors influencing pollen dispersal in the alpine skypilot, Polemonium viscosum (Polemoniaceae). American Journal of Botany 76:419-426.

GALETTO, L. \& BERNARDELLO, G. 2005. Rewards in flowers - Nectar. In Practical Pollination Biology (A. Dafni, P.G. Kevan \& B.C. Husband, eds.). Enviroquest, Ltd. Cambridge, Ontario, p.261-313.

GANDERS, F.R. 1975. Fecundity in distylous and selfincompatible homostylous plants of Mitchella repens (Rubiaceae). Evolution 29:186-188.

GANDERS, F.R. 1979. The biology of heterostyly. New Zealand Journal of Botany 17:607-635.

HAMILTON, C.W. 1990. Variations on a distylous theme in Mesoamerican Psychotria subgenus Psychotria (Rubiaceae). Memoirs of the New York Botanical Garden 55:65-75.

KÉRY, M., MATTHIES, D. \& SPILLMANN, H.H. 2000. Reduced fecundity and offspring performance in small populations of the declining grassland plants Primula veris and Gentiana lutea. Journal of Ecology 88:17-30.

LI, P. \& JOHNSTON, M.O. 2001. Comparative floral morphometrics of distyly and homostyly in three evolutionary lineages of Amsinckia (Boraginaceae). Canadian Journal of Botany 79:1332-1348.

MAL, T.K. \& LOVETT-DOUST, J. 1997. Morph frequencies and floral variation in a heterostylous colonizing weed, Lythrum salicaria. Canadian Journal of Botany 75:10341045.
MARTIN, F.N. 1959. Staining and observing pollen tubes in the style by means of fluorescence. Stain Technology 34:125-128.

MENDONÇA, R.C., FELFILI, J.M., WALTER, B.M.T., SILVA-JÚNIOR, M.C., REZENDE, A.V., FILGUEIRAS, T.S., NOGUEIRA, P.E. \& FAGG, C.W. 2008. Flora vascular do bioma Cerrado: checklist com 12.356 espécies. In Cerrado: ecologia e flora. (S.M. Sano, S.D.P. Almeida \& J.F. Ribeiro, eds.). Embrapa Cerrados/Embrapa Informação Tecnológica, Brasília, v.2, p.421-1279.

PAILLER, T. \& THOMPSON, J.D. 1997. Distyly and variation in heteromorphic incompatibility in Gaertnera vaginata (Rubiaceae) endemic to La Reunion Island. American Journal of Botany 84:315-317.

PAILlER, T., HUMEAU, L. \& FIGIER, J. 1998. Reproductive trait variation in the functionally dioecious and morphologically heterostylous island endemic Chassalia corallioides (Rubiaceae). Biological Journal of the Linnean Society 64: 297-313.

PASSOS, L. \& SAZIMA, M. 1995. Reproductive biology of the distylous Mannetia luteo-rubra (Rubiaceae). Botanica Acta 108:309-313.

PEREIRA, Z.V., VIEIRA, M.F. \& CARVALHO-OKANO, R.M. 2006. Fenologia da floração, morfologia floral e sistema de incompatibilidade em espécies distílicas de Rubiaceae em fragmento florestal do Sudeste brasileiro. Revista Brasileira de Botânica 29:471-480.

REE, R.H. 1997. Pollen flow, fecundity and the adaptive significance of heterostyly in Palicourea padifolia (Rubiaceae). Biotropica 29:298-308.

RICHARDS, J.H. \& KOPTUR, S. 1993. Floral variation and distyly in Guettarda scabra (Rubiaceae). American Journal of Botany 80:31-40.

SOBREVILA, C., RAMIREZ, N. \& ENRECH, N.X. 1983. Reproductive biology of Palicourea fendleri and $P$. petiolaris (Rubiaceae), heterostylous shrubs of a tropical cloud forest in Venezuela. Biotropica 15: 161-169.

STATSOFT, INC. 2001. Statistica (data analysis software system), version 6 . Tulsa.

STONE, J.L. 1995. Pollen donation patterns in a tropical distylous shrub (Psychotria suerrensis; Rubiaceae). American Journal of Botany 82:1390-1398.

SUTHERLAND, S. 1986. Patterns of fruit-set: what controls fruit-flower ratio in plants? Evolution 40: 117-128.

TANNER, E. 1982. Breeding systems in a tropical forest in Jamaica. Biological Journal of the Linnean Society 18:263-278.

TAYLOR, C.M. 1997. Conspectus of the genus Palicourea (Rubiaceae: Psychotriae) with the description of the some new species from Ecuador and Colombia. Annals of the Missouri Botanical Garden 84:224-262. 
TEIXEIRA, L.A.G. \& MACHADO, I.C. 2004. Biologia da polinização e sistema reprodutivo de Psychotria barbiflora DC. (Rubiaceae). Revista Brasileira de Botanica 18:853-862.
WASHITANI, I., OSAWA, R., NAMAI, H. \& NIWA, M. 1994. Patterns of female fertility in heterostylous Primula sieboldii under severe pollinator limitation. Journal of Ecology 85:571-579. 